\title{
Diacronie
}

Studi di Storia Contemporanea

$N^{\circ} 19,3 \mid 2014$

Miscellaneo

\section{Esporre per costruire: un'analisi storico-tipologica di alcuni grandi eventi come momento di ridefinizione identitaria}

Maria Elena Buslacchi

\section{(2) OpenEdition \\ Journals}

Edizione digitale

URL: http://journals.openedition.org/diacronie/1555

DOI: 10.4000/diacronie. 1555

ISSN: 2038-0925

Editore

Association culturelle Diacronie

Notizia bibliografica digitale

Maria Elena Buslacchi, «Esporre per costruire: un'analisi storico-tipologica di alcuni grandi eventi come momento di ridefinizione identitaria », Diacronie [Online], № 19, 3 | 2014, documento 1, Messo online il 01 septembre 2014, consultato il 20 avril 2019. URL : http://journals.openedition.org/ diacronie/1555; DOI : 10.4000/diacronie.1555 


\section{Diacronie}

\section{Esporre per costruire: un'analisi storico- tipologica di alcuni grandi eventi come momento di ridefinizione identitaria}

Maria Elena BUSLACCHI*

Con questo articolo mi propongo di analizzare da diverse prospettive la storia e lo sviluppo di alcuni grandi eventi, come le Esposizioni Internazionali e le Capitali Europee della Cultura. Situandoli nel dibattito teorico ed osservando alcuni esempi concreti, questo lavoro mostrerà come le politiche di city-marketing e di competizione fra territori abbiano portato ad un progressivo svuotamento di significato dei titoli attribuiti e ad un collegamento sempre più evidente con la rigenerazione urbana. Le differenze tipologiche tra gli eventi sfumano: il loro carattere strumentale è prevalente dev'essere collegato ad altri parametri, tra i quali la dimensione rituale non ha perso la sua importanza. Gli eventi sono diventati una vera e propria strategia delle amministrazioni locali, i cui effetti non ricadono solo su un'élite ristretta, ma sulla comunità intera.

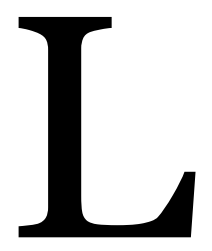

'evoluzione della società in una forma sempre più globalizzata ed interconnessa, detta "dei flussi"1 o "liquida"2 sembrerebbe rendere superflui i momenti di incontro tradizionale. Eppure, così come gli stessi teorici di questa evoluzione hanno evidenziato, l'economia capitalista ha rafforzato il ruolo delle città e reso importante la scansione del tempo secondo periodici momenti di ripensamento dell'identità urbana nel suo complesso. Chiamare a raccolta

\footnotetext{
${ }^{1}$ CASTELLS, Manuel, L'ère de l'information, La société en réseaux, Paris, Fayard, 1998.

2 BAUMAN, Zygmunt, Modernità liquida, Roma-Bari, Laterza, 2006.
} 
non soltanto la cittadinanza, ma anche un pubblico esterno, ancorché magari settoriale, impone di rimettere in discussione la struttura materiale e l'immagine simbolica con cui ogni città si presenta a se stessa e al mondo. La calendarizzazione di questi momenti è diventata, nel corso del XX secolo e in maniera quasi esasperata nel XXI, una vera e propria strategia per l'amministrazione della città, che affida non soltanto le grandi opere, ma sempre più anche il mantenimento ordinario ad occasioni straordinarie di disponibilità finanziaria. L'eccezionale afflusso di capitali dato dall'intervento di organismi istituzionali di competenza più estesa (lo Stato, l'Unione Europea) e dalla compartecipazione di attori privati (sponsor, mecenati, associazioni) permette di realizzare opere di cui la città avvertiva spesso già l'esigenza in precedenza, ma che risultavano inattuabili con i mezzi dell'amministrazione ordinaria, nella nuova configurazione economica occidentale. L’importanza di questi momenti e il loro nesso con la gestione delle questioni urbane non più solo eccezionali, ma quotidiane porta ad una serrata competizione tra città per il loro ottenimento sotto la forma, essenzialmente, del grande evento.

Questa politica di marketing urbano 3 è tesa, come in un circolo, ad attirare investimenti che migliorino l'immagine della città la quale, a sua volta, sarà in grado di attirare nuovi investimenti. In questa dinamica entra in gioco anche il tentativo di attrarre sulla città una popolazione di reddito medio-alto: come è comune sentirsi ripetere dagli amministratori locali, "Con una popolazione ricca condivideremo la ricchezza; con una popolazione povera condivideremo la povertà". Allo stesso modo in cui cercano di attirare un grande evento, quindi, le diverse Direzioni delle amministrazioni insistono per far insediare sul proprio territorio aziende in grado di portare lavoro e disponibilità economica. In questo senso, la possibilità di offrire servizi efficienti e, più generalmente, spazi gradevoli da abitare rende più appetibile la città.

Nel discorso politico questi diversi aspetti sono spesso confusi in una stessa retorica del cambiamento, che si serve di strumenti diversi, su più livelli, per lo stesso fine. Proprio in virtù della loro compenetrazione in fase gestionale, l'ordinario e l'extraordinario si confondono e rendono difficile la distinzione delle singole politiche. Di riflesso, anche la letteratura sul tema, come osserva Guala ${ }^{4}$, ha spesso trattato in

3 Per una sua teorizzazione si vedano ad esempio: AMENDOLA, Giandomenico, La città postmoderna: magie e paure della metropoli contemporanea, Bari, Laterza, 1997; VAN DER BERG, Leo, BRAUN, Eric, «Urban Competitiveness, Marketing and the Need for Organising Capacity», in Urban Studies, 36, 1999, pp. 987-999; KAVARATZIS, Mihalis, "From City Marketing to City Branding: Towards a Theoretical Framework for Developing City Brands», in Place Branding, 1, 1/2004, pp. 58-73.

4 GUALA, Alessandro Chito, «Per una tipologia dei mega-eventi», in Bollettino della Società Geografica Italiana, serie XII, VII, 4/2002, pp. 861-894. 
termini simili i cosiddetti mega events, big events, hallmark events, special events. Per quanto diversi nelle loro intenzioni, nel loro pubblico, nel tipo di capitali che mobilitano, questi eventi sono spesso accomunati proprio perché oggetto di una strategia diffusa che li vede indistintamente come strumenti della rigenerazione della città e della sua immagine.

Nella necessità analitica di osservare le differenze tra questi aspetti innanzitutto è necessario separare un evento da una politica, o da una tendenza generale. Un evento, qualunque sia l'utilizzo che, strumentalmente, se ne fa, è innanzitutto un momento non ordinario di aggregazione tra individui, intenzionale e programmato, circoscritto nel tempo e nello spazio, dedicato o intitolato ad un tema.

Gli studi di Ritchie 5 e Hall ${ }^{6}$ hanno da tempo evidenziato come l'impatto di un evento possa esprimersi in ambito economico, turistico e commerciale, fisico, socioculturale, psicologico e politico. Sulla base di queste considerazioni e di come possa variare, per ciascuno di questi parametri, l'influenza di un evento su un territorio, Roche ${ }^{7}$ propone una categorizzazione che ha valenza non soltanto teorica, ma anche di riconoscimento di alcune tassonomie vigenti: ad esempio, quella operante nelle politiche del Comitato Olimpico Internazionale ${ }^{8}$. Nella categoria dei mega-events sono quindi inseriti soltanto quegli eventi che hanno un pubblico e una diffusione mediatica globale: le esposizioni universali, le Olimpiadi, i mondiali di calcio. Seguono gli special events: ad esempio il Gran Premio di Formula Uno e altri campionati sportivi che, pur giocandosi su scala anche mondiale, interessano un pubblico più settoriale. Gli hallmark events hanno per lo più portata nazionale: in essi rientrano i festival più celebri e le manifestazioni sportive del calibro del Giro d'Italia o del Tour de France. Infine, i community events sono celebrazioni strettamente legate alla comunità locale che vi partecipa, meno caratterizzati da un contenuto forte e più significativi per la loro funzione di aggregazione del gruppo sociale che ne è al tempo stesso organizzatore, pubblico e protagonista.

La tassonomia di Roche privilegia sicuramente gli eventi sportivi sugli altri e lascia insolute diverse questioni. Nella sua critica a questo modello, Guala ${ }^{9}$ propone una categorizzazione più precisa, che include anche le fiere specializzate (ad esempio del

5 RITCHIE, John Raymond Brent, «Assessing the impact of hallmark events», in Journal of Travel Research, 23, 1/1984, pp. 2-11.

${ }^{6}$ HALL, Colin Michael, Hallmark Tourist Events: Impacts, Management and Planning, London, Belhaven Press, 1992.

7 ROCHE, Maurice, Mega-Events and Modernity: Olympics and Expos in the Growth of Global Culture, London, Routledge, 2000.

8 Si veda INTERNATIONAL OLYMPIC COMMITTEE, Olympic Marketing, 1998 Fact File, Lausanne, IOC, 1998

9 Ibidem. 
libro, della nautica), i summit internazionali (come il G7, il G8, il Forum delle Culture), le grandi mostre, le reti di città (della Cultura, smart cities, città interculturali, città educative...), gli eventi religiosi (come i giubilei) e persino le grandi opere (il Guggenheim Museum a Bilbao, il Millennium Dome a Londra, la riqualificazione dei waterfront). Gli eventi sono suddivisi per contenuto, ordine di grandezza e copertura televisiva (diretta o servizi). In questo quadro la nozione stessa di evento sbiadisce, potendo comprendere, da un lato, l'inserimento di una città in un network che non necessariamente prevede momenti di grande aggregazione e, dall'altro, interventi di rigenerazione urbana.

La classificazione di Guala mette in realtà in luce un progressivo accostamento delle diverse tipologie di evento che, nascendo in contesti assai disparati, finiscono per assumere caratteristiche simili, tanto da obbligare ad una loro ripartizione analitica. Questi eventi si assomigliano sempre di più perché hanno vissuto un'evoluzione parallela: da un lato hanno subito un processo di erosione dei contenuti che li porta ad essere di volta in volta "riempiti" con un tema diverso, dall'altro si sono legati sempre di più ad operazioni urbanistiche imponenti.

\section{La funzione rituale dell'evento}

La dimensione rituale del grande evento non è certo nuova, anzi, come osserva Roche, lo caratterizza fin dall'inizio:

L'organizzazione di grandi eventi internazionali era e resta importante nella "storia di una nazione", di un popolo, di un paese. Essi rappresentavano infatti, e continuano a rappresentare, momenti chiave in cui le nazioni possono costruire e mostrare immagini di sé per un riconoscimento nei confronti delle altre nazioni e "agli occhi del mondo". Essi rappresentavano, e continuano a rappresentare, inoltre, momenti chiave in cui si può inventare e immaginare la "tradizione" e la "comunità" nazionale, ivi compresi il passato, il presente e il futuro (il progresso nazionale, il potenziale e il "destino") e non solo da e per i leader e i cittadini della nazione ospite, ma anche da e per il pubblico di altre nazioni ${ }^{10}$.

${ }^{10}$ Ibidem. 
Questa dimensione è assai evidente nelle esposizioni ${ }^{11}$ legate agli anniversari di momenti fondativi della storia di una nazione: nel 1876 l'esposizione di Philadelphia celebra il centenario dell'indipendenza degli Stati Uniti, nel 1889 quella di Parigi onora la Rivoluzione Francese, Liegi nel 1905 ospita l'esposizione per il $75^{\circ}$ anniversario dell'indipendenza del Belgio, nel 1961 è la volta di Torino per il centenario dell’Unità d'Italia. Al di fuori del circuito delle esposizioni universali, in tempi più recenti, Torino festeggia i 150 anni dell'Unità d'Italia non a caso ristrutturando ed aprendo al pubblico un'area simbolicamente importante per l'identità urbana e nazionale: le Officine Grandi Riparazioni della Fiat.

Come osserva Dell'Osso ${ }^{12}$, le esposizioni universali hanno subito, nel corso della loro storia, una traslazione contenutistica che andava dall'esaltazione del progresso, dell'industria e della tecnologia delle prime manifestazioni (da Londra 1851 a New York 1939) ad un momento di incontro e scambio culturale più ampio. Questa apertura, tuttavia, continuava a considerare la cultura prioritariamente in senso tecnico e proprio la riduzione del sapere alla sua dimensione pratica non lasciava ampio spazio all'aspetto artistico o, più in generale, umanistico. Per questo alle esposizioni universali come momenti d'incontro tra le nazioni hanno presto fatto seguito le esposizioni internazionali in ambito artistico, non riconosciute dal BIE: tra le prime e più importanti vi è la Biennale di Venezia, concepita per iniziativa dell'amministrazione comunale nel 1983 e tenutasi per la prima volta nel 1895. Alle arti intese come "mestieri", che ritroviamo con frequenza nelle intitolazioni delle prime esposizioni universali ${ }^{13}$, si affiancano le arti "decorative" o le "belle arti", il cui aspetto funzionale è controverso: a Parigi si tiene nel 1925 l'Esposizione Universale delle Arti decorative e industriali moderne, cui prendono parte, emblematicamente, da un lato Le Corbusier, con la sua cellule d'habitation, dall'altro Pablo Picasso, Férnand Léger, con le loro opere pittoriche d'avanguardia.

\footnotetext{
${ }^{11}$ Spesso, nel linguaggio comune, le esposizioni universali e internazionali sono accomunate in una terminologia unica. In realtà la classificazione del BIE è precisa ed è cambiata più volte nel tempo: ci atterremo qui, per semplicità, alle esposizioni riconosciute come «World Expos», la cui denominazione ufficiale dal 1996 è "International Registered Exhibitions" e corrisponde a «the largest and broadest among mega events. The size of the site is unlimited and participants build their own pavilions. World Expos are also referred to as Universal Expos because the chosen theme concerns the whole of humanity» (BIE 1996).

12 DELL'OSSO, Roberto Expo, Le Esposizioni Universali da Londra 1851 a Shanghai 2010. Milano, Clup, 2006.

13 A Parigi nel 1855 si tiene l'esposizione "Agricoltura, industria e arti”, a Londra nel 1862 "Industria e arti", ancora a Parigi nel 1867 "Agricoltura, industria e arti" e nel 1878 "Agricoltura, arte e industria", a Melbourne nel 1880 "Arti, manufatti, prodotti industriali e agricoli di ogni nazione". Il termine "arte" sparisce poi dalle intitolazioni fino al 1937, con l'esposizione di Parigi "Arte e tecnica nella vita moderna" e si riscontra poi assai raramente negli anni successivi.
} 
Il concetto di arti "applicate" si ritrova chiaramente nella sola esposizione di stampo artistico riconosciuta dal BIE: la Triennale di Milano. Nata nel 1923 a Monza come la prima Biennale delle arti decorative e simbolicamente collocata, a partire dal 1933, a Palazzo dell'Arte a Milano, questa esposizione ha per fine di avvicinare i mondi dell'industria, della produzione e dell'arte. La Triennale si tiene più o meno regolarmente fino al 1996.

Un caso a parte è quello delle Esposizioni Coloniali: il loro intento celebrativo passa anche attraverso l'esposizione dell'arte e della cultura "esotica" dei popoli colonizzati: in questo senso, però, l'arte fine a se stessa è presentata in opposizione all'arte “civilizzata”, funzionale alla società.

Quando, nel 1985, l'attrice greca e ministro della Cultura Melina Mercouri lancia il progetto delle Città (poi Capitali) Europee della Cultura, lo scopo è proprio quello di celebrare un'identità comune europea, fondata su una cultura condivisa. In opposizione non soltanto di competenza geografica e politica, ma anche contenutistica, con le esposizioni più diffuse, nel discorso inaugurale dell'iniziativa dell'Unione Europea Mercouri proclama quindi che «È tempo che la voce dei nostri ministri della cultura si senta forte quanto quella dei tecnocrati. La cultura, l'arte e la creatività non sono meno importanti della tecnologia, del commercio e dell'economia» ${ }^{14}$. Con l'ambizione di raccogliere il meglio dell'arte e della cultura in ambito europeo e di celebrare, in senso storico o progettuale, l'appartenenza ad una stessa comunità ${ }^{15}$, Atene è quindi la prima città a ricevere questo titolo e ad inaugurarne la storia con spettacolari fuochi d'artificio sull'Acropoli. Seguono Firenze nel 1986, Parigi nel 1989: l'identità europea si tesse a poco a poco integrando il contributo di ogni nazione ed ogni epoca. Le Capitali europee della Cultura diventano, da «extended summer festival» ${ }^{16}$, eventi che durano un anno intero e che coinvolgono il mondo artistico, culturale ed anche economico del territorio.

Quel che Roche osserva per la dimensione nazionale si traspone allora facilmente su altri livelli: quello sovranazionale, che legittima il progetto politico dell'Europa attraverso la ricostruzione delle sue origini "culturali"; quello transnazionale, delle élites appartenenti a diversi mondi (dell'arte, dell'architettura...) e infine quello urbano, dal momento che proprio questa scala diventa rilevante nella nuova configurazione economica, politica e culturale della società contemporanea. La città, portavoce della sua regione e della nazione a cui appartiene, gareggia con gli altri hub possibili ed è

\footnotetext{
14 EUROPEAN COMMISSION, European capitals of culture. The road to success : from 1985 to 2010, Bruxelles, European Commission Publications, 2009.

${ }_{15}$ CORIJN, Eric, VAN PRAET, S., Antwerp 93 in the context of European Cultural Capitals: art policy as politics, Bruxelles, Vrije Unversiteit Brussel, 1994.

${ }_{16}$ RICHARDS, Greg, Cultural Tourism in Europe, Wallingford, CABI, 1996.
} 
pronta a ripensarsi assumendo caratteristiche nuove, inattese, purché funzionali a nutrirla.

\section{L'evento e la rigenerazione urbana}

Solo un grande evento può giustificare una grande trasformazione $o$, da un'altra prospettiva, una grande trasformazione finisce per diventare - come osservava Guala un grande evento.

Il clima di generale competizione induce le amministrazioni a cercare di attrarre investimenti sul loro territorio proprio nell'ottica di migliorare l'immagine della città ${ }^{17}$. In questo senso la strategia operativa è spesso quella di creare un'icona ex novo: le esposizioni storiche investivano principalmente aree che si ritenevano, a ragione o a torto, urbanisticamente vuote - non costruite o in condizioni malsane o degradate; $\mathrm{i}$ grandi eventi odierni tendono a legarsi al recupero di aree abbandonate, dismesse e si inseriscono nella configurazione urbana postindustriale. L'evento prende forma così in zone che almeno nel vicino passato non facevano parte della città. Sono proprio queste aree a diventarne il nuovo simbolo, a modificarne, attraverso una grande copertura mediatica, il posizionamento sullo scenario internazionale.

L'evento ha luogo in città, ma ne cambia contemporaneamente la mappa, creando nuovi spazi e proiettandosi ben al di là del proprio finito orizzonte temporale. Il suo ciclo di vita si estende quindi in un arco di tempo piuttosto lungo, che va ad interferire con la dimensione della vita quotidiana degli abitanti. Proprio per questo, come osserva Amendola ${ }^{18}$, non si può più misurare l'impatto di un evento soltanto sulla base di parametri tradizionali, quali il numero di visitatori o la copertura mediatica. Se è vero che l'entità dei finanziamenti erogati alle città da agenti esterni varia notevolmente a seconda che si parli di un'Olimpiade estiva, un'Olimpiade invernale, un'esposizione universale, una Capitale europea della Cultura, è altresì vero che l'evento ha sempre luogo in un contesto di relazioni e di strategie più ampio, che chiama in causa progetti (e finanziamenti) complementari e paralleli.

La cosiddetta culture-led regeneration, rigenerazione urbana attraverso i grandi eventi, si è affermata non soltanto perché questi apportano alla città un afflusso di denaro straordinario, ma proprio per l'alto valore simbolico dell'evento in sé. La dimensione rituale dell'evento impone un ripensamento della città nel suo complesso e

17 VICARI HADDOCK, Serena, Brand-Building. The Creative City. A Critical Look at Current Concepts and Practices, Firenze, Firenze University Press, 2010.

18 Ibidem. 
permette di concepire - e spesso, da parte delle amministrazioni, far accettare alla popolazione - trasformazioni e cambiamenti altrimenti difficili da realizzare.

Il caso delle Capitali europee della cultura è emblematico: dal 1999 la rigenerazione urbana è inserita esplicitamente negli obiettivi dell'operazione, già precedentemente "sfruttata" in tal senso. Il caso più eclatante fu Glasgow 199019: considerata come una delle Capitali europee della Cultura di maggior successo, la città aveva coronato con l'attribuzione del titolo un processo di rigenerazione urbana già avviato nel corso degli anni Ottanta (Glasgow Miles Better) e portato a termine proprio per quel traguardo. Molte Capitali europee della Cultura sono andate in questa direzione, seguendo in maniera più o meno diretta l'esempio di Glasgow: Luxembourg 1995 inaugura in occasione dell'evento il nuovo Museo di arte moderna e nuova Filarmonica, Copenhagen 1996 lavora sll'area portuale, Weimar 1999 rinnova la stazione ferroviaria, Tessalonica 1997 e Porto 2001 ristrutturano in profondità la città.

A fare scuola è soprattutto Barcellona, che nel 1992 ospita le Olimpiadi e rinnova le aree portuali e industriali dismesse: la rivoluzione, urbanistica e d'immagine, è tale da assurgere a modello e da essere costantemente citata come esempio di strategia di successo.

Con il Report di Palmer \& Rae (2004) sulle Capitali europee della Cultura, che mette in luce il nesso tra attribuzione del titolo e rigenerazione urbana, la politica di scelta delle città da designare Capitali passa dall'elezione di centri riconosciuti della vita culturale internazionale a città più note per i loro problemi sociali che per la loro attività culturale (quali Liverpool nel 2008, Essen nel 2010, Marsiglia nel 2013). Il "rule book" dell'evento insiste sulla dimensione identitaria europea, sulla partecipazione dei cittadini e sull'effetto a lungo termine:

As regards 'City and Citizens' the programme shall: (a) foster the participation of the citizens living in the city and its surroundings and raise their interest as well as the interest of citizens from abroad; (b) be sustainable and be an integral part of the long-term cultural and social development of the city ${ }^{20}$.

19 Per una trattazione del caso di Glasgow 1990 si vedano GARCIA, Beatriz, «Urban regeneration, arts programming and major events: Glasgow 1990, Sydney 2000 and Barcelona 2004", in International Journal of Cultural Policy, 10, 2004, pp. 103-116; GARCIA, Beatrizm «Deconstructing the City of Culture: The Long-term Cultural Legacies of Glasgow 1990», in Urban Studies, 42, 2005, pp. 841-868; MOONEY, Gerry, «Cultural Policy as Urban Transformation? Critical Reflections on Glasgow, European City of Culture 1990", in Local Economy, 19, 4/2004, pp. 327-340; MYERSCOUGH, John, Monitoring Glasgow 1990, Glasgow, Glasgow City Council-Strathclyde Regional Council and Scottish Enterprise, 1991. ${ }^{20} 1622 / 2006 / \mathrm{EC}$. 
Di che tipo di sviluppo si parli è ulteriormente specificato (EC 2009: 10):

A key challenge for European Capitals of Culture is to ensure that the project is embeded as part of a long-term political commitment and strategy by the city to using culture to develop itself into - and to remain - a creative city.

L'accezione di cultura, allora, si fa più indefinita: oscilla tra l'esaltazione del passato - di una cultura comune - e quella della modernità - di una nuova comunità politica. Si trasforma in motore del cambiamento capace di giustificare operazioni urbanistiche massicce e profondi stravolgimenti dell’identità urbana. "Creative city" è la qualificazione che, a partire dalle definizioni di Landry ${ }^{21}$ e Florida ${ }^{22}$ e non di rado secondo loro fantasiose interpretazioni localistiche, identifica nel dibattito internazionale le città che si incentrano su un'economia postindustriale, dei servizi, della cultura e del turismo. Sostenere il cambiamento a lungo termine in questo senso significa promuovere, al livello delle città europee, un preciso modello di sviluppo competitivo.

Proprio mentre accentua l’importanza della strategia a lungo termine ed accompagna gli interventi di rigenerazione, il titolo di Capitale europea della Cultura non si accompagna tuttavia ad un sostegno finanziario massiccio: alle città designate Capitali Europee della Cultura è attribuito dal 2007 un kick-off fund di 1,5 milioni di euro, poca cosa in confronto al budget complessivo di ogni singola operazione, che oscillava tra gli 8 e i 74 milioni negli anni precedenti, con una media di $37^{23}$.

La portata dei finanziamenti provenienti dall'Unione Europea, tuttavia, non smentisce l'importanza dell'evento, ma va nella direzione dell'integrazione sul territorio. Proprio per la sua dimensione rituale, di ripensamento della città nel suo complesso, la manifestazione ha la capacità di coinvolgere un gran numero di attori pubblici e privati nell'ambito del territorio diffuso che in quel momento investe. L'attivazione simultanea di soggetti diversi, che si coordinano e compartecipano alla strutturazione dell'evento, mette a disposizione della città una quantità di capitali altrimenti difficile da mettere in campo e permette la realizzazione di opere altamente significative per la sua ridefinizione d'immagine.

${ }^{21}$ LANDRY, Charles, BIANCHINI, Franco, The Creative City, London, Demos, 1995.

${ }^{22}$ FLORIDA, Richard, The Creative Class: And How it's Transfroming Work, Leisure, Community and Everyday Life, New York, Basic Books, 2002.

23 PALMER, Robert, European Cities and Capitals of Culture: Study Prepared for the European Commission, Brussels, Palmer-Rae Associates, 2004. 
La costruzione di una rete di istituzioni che agiscono in maniera coordinata spesso - anche se non automaticamente - sopravvive al ciclo di vita dell'evento e tende a prefigurare l'opportunità di sfruttare nuove occasioni di visibilità e di investimento straordinario. L'evento, così, diventa uno strumento della strategia del quotidiano.

\section{L'evento come contenitore}

L'evento si fa contenitore perché la sua valenza è sempre più funzionale: serve alla comunità di riferimento come momento rituale di riaffermazione di fronte a sé e agli altri e fornisce i mezzi necessari a realizzare opere spesso già concepite e attese.

Se l'amministrazione di una città fonda sempre di più la sua gestione ordinaria su eventi straordinari ricorrenti, il contenuto di questi eventi sbiadisce, poiché secondario. È importante aprire una strada, ristrutturare un'area industriale dismessa: spesso non è così rilevante, nell'ottica dell'amministrazione - o della cordata delle istituzioni - che i fondi per realizzare i lavori siano messi a disposizione con l'occasione di una competizione sportiva, di una manifestazione culturale o con una fiera tecnologica. L'obiettivo con cui si cerca l'evento e si avviano le procedure di candidatura di una città è sempre meno legato alla sua specificità e sempre più dipendente dal bisogno di visibilità che le città acquisiscono nel generale clima di competizione tra territori. I diversi eventi, categorizzati per mobilitazione di fondi e copertura della stampa, diventano potenziali risposte alle esigenze delle città nella strutturazione, fisica e simbolica, di un'immagine nuova in senso generale o mirato su specifiche problematiche.

Dal canto loro, gli eventi - nella fattispecie gli enti e i comitati transnazionali che li concepiscono - si moltiplicano, si fanno più versatili e disponibili ad essere adattati alle specifiche esigenze. Nei rule books questa esigenza va definendosi in maniera sempre più chiara negli anni e il minimo comune denominatore delle manifestazioni si assottiglia progressivamente: come osserva il segretario del Bureau International des Expositions, Gonzales Loscertales, a proposito delle esposizioni universali,

Innovation is the hallmark of Expos. Whether it is architecture, urban planning, new services, exhibition content, performance, new technologies and so on, each Expo is built and showcases inventions and novel approaches ${ }^{24}$.

24 GONZALEZ LOSCERTALES, Vicente, A pillar of the success of EXPO 2010: the Theme Development, Paris, BIE, 2011. 
"Expo" sembra quindi equivalere a "innovazione", elemento residuale di un apparato più complesso di concetti operanti nelle esposizioni internazionali e universali "storiche", che in qualche modo incarnavano la modernità stessa, nel loro promuovere i valori del progresso, della tecnica, del commercio, dell'universalità. L'evento diventa il contenitore di un tema specifico, di volta in volta stabilito ${ }^{25}$ : a concetti assai generali come l'industria (Londra 1851 e 1862, Parigi 1855, 1867, 1878), il bilancio di un secolo o del "mondo" (Parigi 1900, Chicago 1933, Bruxelles 1958, Monréal 1967, Osaka 1970), si vanno a sostituire tematiche più specifiche quali l'età delle scoperte (Siviglia e Genova 1992), il rapporto tra uomo, ambiente e tecnologia (Hannover 2000), la città (Shanghai 2010) fino a "Nutrire il pianeta, Energia per la vita" (Milano 2015).

Del concetto di esposizione universale non resta che una traccia:

The Expo is the canvas and the theme is the core vision that supports the masterplan of the Expo ${ }^{26}$.

Un'evoluzione simile vivono le Capitali europee della Cultura. Come osserva Sassatelli, nonostante la crescente importanza del titolo e delle sue concrete realizzazioni, l'iniziativa dell'Unione Europea si è sviluppata attraverso un modello minimale:

The programmes set up by cities are increasingly complex and ambitious, entailing initiatives that would not easily fit into a traditional definition of cultural policy and that clearly aim at a wide notion of culture. This success may arguably be linked also to the ability of the EU to mantain a low profile, thus avoiding the feeling that the programme is a top-down invasion of the delicate sphere of cultural identities. But these sphere is, of course, precisely what the ECOC is targeted at 27.

Le molteplici interpretazioni date del titolo, da quelle che accentuano la dimensione europea, spesso nelle Capitali dell'Est, a quelle che quasi la dimenticano, rimarcando

\footnotetext{
25 «The BIE embodies the international community that, when selecting the host city or accepting to participate, takes into very high consideration the relevance of the theme. It must be powerful enough to inspire the development of attractive pavilion content and to enable the participants to show some of the outstanding achievements or their country. [...] By placing a greater emphasis on the theme as the central core and organizing principle of Expos, the BIE intends to strengthen their role as instruments for public diplomacy and for international public education».

${ }^{26}$ Ibidem

${ }^{27}$ SASSATELLI, Monica, The Logic of Europeanizing Cultural Policy, in MEINHOF, Ulrike, TRIANDAFYLLIDOU, Anna (eds), Transcultural Europe. London, Palgrave, 2006, pp. 24-42.
} 
piuttosto l'importanza del termine "cultura", rendono la Capitale europea della Cultura un «floating signifier» 28 .

\section{Conclusioni}

In ragione della loro dimensione rituale, del legame con la rigenerazione urbana e dello svuotamento contenutistico gli eventi vanno assomigliandosi sempre di più: una categorizzazione basata su finanziamenti, pubblico, copertura mediatica risulta allora sempre più angusta. Sempre più significativo è invece l’inserimento in una strategia complessiva a livello territoriale e la capacità di inquadrare l'evento come un'occasione straordinaria di ridefinizione dell'identità locale su micro- e macro-scala. In questo quadro acquisiscono importanza quelle città che riescono ad inserirsi non soltanto nelle reti commerciali e finanziarie globali, ma anche nei circuiti della vita artistica, culturale e intellettuale internazionale. Ad avere maggior successo possono essere allora anche città di dimensioni medio-piccole che costituiscono, però, un punto di riferimento per la "società dei flussi" e in cui la capacità di impatto di un evento è da valutarsi non soltanto in termini assoluti, ma relativi al contesto. Un titolo è capace di incidere in maniera più forte su un territorio pre-disposto ad accoglierlo e in proporzione al suo impatto non soltanto economico, ma simbolico: come osserva Greg Richards a proposito delle Capitali europee della cultura già a quindici anni dalla loro istituzione,

The event has become so popular with policy-makers that bids to stage the event have taken on the same kind of competitive element only usually seen for the Olympic Games nomination²9.

Le classificazioni in tipologie di evento fino ad oggi proposte, quindi, mantengono la loro pregnanza in quanto parametri di analisi da considerare in un sistema più ampio, in cui l'evento è diventato una strategia di amministrazione ordinaria dei territori e in cui gli attori locali sono sempre più consapevoli del loro ruolo nella ridefinizione identitaria - fisica e simbolica - del loro contesto urbano e metropolitano.

28 PALONEN, Emilia, «Multi-Level Cultural Policy and Politics of European Capitals of Culture», in Nordisk kulturpolitisk tidskrift, 1/2010, pp. 87-108.

${ }^{29}$ RICHARDS, Greg, «The European Cultural Capital Event: Strategic Weapon in the Cultural Arms Race?», Journal of Cultural Policy, 6, 2/2000, pp. 159-181. 


\section{* L'autore}

Maria Elena Buslacchi è dottoranda in Storia - Antropologia presso l'Università degli Studi di Genova e l’École des Hautes Études en Sciences Sociales - Centre Norbert Elias di Marsiglia. Il suo progetto di ricerca analizza in maniera comparativa e pluridisciplinare le politiche e gli effetti di due Capitali Europee della Cultura: Genova 2004 e Marsiglia 2013. Fa parte dell'équipe di ricerca MP2013-Publics et Pratiques Culturelles e del Laboratorio di Studi Urbani "Incontri in Città" del DAFiSt - Unige.

URL: < http://www.studistorici.com/progett/autori/\#Buslacchi >

\section{Per citare questo articolo:}

BUSLACCHI, Maria Elena, «Esporre per costruire: un'analisi storico-tipologica di alcuni grandi eventi come momento di ridefinizione identitaria», Diacronie. Studi di Storia Contemporanea, 29/09/2014, URL:<http://www.studistorici.com/2014/09/29/buslacchi_numero_19/ >

\section{Diacronie Studi di Storia Contemporanea $\beta$ www.diacronie.it}

Risorsa digitale indipendente a carattere storiografico. Uscita trimestrale. redazione.diacronie@hotmail.it

Comitato di redazione: Jacopo Bassi - Luca Bufarale - Elisa Grandi - Deborah Paci - Fausto Pietrancosta - Matteo Tomasoni - Luca Zuccolo 